\title{
PARK2 negatively regulates the metastasis and epithelial-mesenchymal transition of glioblastoma cells via ZEB1
}

\author{
HAIYANG WANG, ZHENFENG JIANG, MENG NA, HAITAO GE, \\ CHONGYANG TANG, HONG SHEN and ZHIGUO LIN \\ Department of Neurosurgery, The First Affiliated Clinical Hospital of Harbin Medical University, \\ Harbin, Heilongjiang 150001, P.R. China
}

Received May 16, 2016; Accepted April 24, 2017

DOI: $10.3892 / 01.2017 .6488$

\begin{abstract}
Glioblastoma multiforme (GBM), one of the most aggressive human malignant brain tumors, is induced by multiple complex pathological mechanisms. The main cause of mortality in patients with GBM is the invasion-metastasis cascade of tumor cells. The dysfunction of Parkinson protein 2 E3 ubiquitin protein ligase (PARK2) is closely linked with the development of certain human cancers. However, whether PARK2 is associated with metastasis in GBM remains unknown. The present study demonstrated that the metastasis and invasion of U87 cells were significantly repressed by PARK2 overexpression. Conversely, knockdown of PARK2 facilitated the metastasis and invasion of A172 cells. Furthermore, PARK2 downregulated zinc finger E-box-binding homeobox 1 (ZEB1) expression and mitigated epithelial-mesenchymal transition (EMT). Promoter effects of PARK2 knockdown on cell metastasis and EMT were antagonized by silencing ZEB1 expression. These results indicated that PARK2 participated in regulating the invasion-metastasis cascade of cancer cells by depressing ZEB1 expression and acting as a metastasis suppressor in GBM progression, providing a potential therapeutic approach for GBM treatment.
\end{abstract}

\section{Introduction}

Glioblastoma multiforme (GBM), one of the most aggressive human malignancies, is a brain cancer that originates from glial cells (1). GBM is characterized by diffuse infiltration of the brain tissue surrounding the bulk of the tumor $(2,3)$. The standard treatment option is typically surgical resection

Correspondence to: Dr Zhiguo Lin, Department of Neurosurgery, The First Affiliated Clinical Hospital of Harbin Medical University, 23 Youzheng Street, Harbin, Heilongjiang 150001, P.R. China E-mail: zhiguolinqa@tom.com

Key words: glioblastoma, Parkinson protein 2 E3 ubiquitin protein ligase, metastasis, zinc finger E-box-binding homeobox 1, epithelial-mesenchymal transition followed by radiotherapy and chemotherapy (4). Due to the highly diffuse infiltration, achieving complete surgical resection is impractical and the efficiency of radiotherapy is reduced. Thus, examining the mechanisms that affect the invasive behavior of glioma cells may help to establish novel effective therapies and develop novel treatment strategies.

Parkinson protein 2 E3 ubiquitin protein ligase (PARK2) is a key factor in the regulation of the development of numerous diseases, including multiple human malignancies (5). Previous studies have demonstrated that PARK2 deficiency promotes the initiation of colorectal adenoma and hepatocellular carcinoma and accelerates the progression of tumorigenesis $(6,7)$. Conversely, PARK2 overexpression mitigates cell proliferation and suppresses the progression of breast and lung cancer cell cycles $(8,9)$. Although somatic alterations of PARK2 are frequently observed in GBM cells (10), the consequences of inactivating PARK2 in the invasion-metastasis cascade of glioma cells remain to be fully understood. Therefore, the function of PARK2 in the metastasis of GBM cells and the corresponding molecular mechanisms require further assessment.

The present study revealed that PARK2 overexpression mitigated the metastasis and invasion of GBM cells, while PARK2 knockdown promoted the invasion-metastasis cascade of GBM cells. Furthermore, PARK2 negatively regulated the expression of zinc finger E-box-binding homeobox 1 (ZEB1). The promoter effects of PARK2 knockdown on metastasis and epithelial-mesenchymal transition (EMT) were attenuated by silencing ZEB1 expression. These results revealed an important mechanism underlying the regulation of the invasion-metastasis cascade of GBM cells, which may be a potential treatment target for GBM.

\section{Materials and methods}

Cell line preparation and culture. U87, U251, U373, A172 and LN444 were obtained from the American Type Culture Collection (Manassas, VA, USA). All cell lines were maintained in Dulbecco's modified Eagle's medium (DMEM; Gibco; Thermo Fisher Scientific, Inc.) and supplemented with $10 \%$ fetal bovine serum (FBS; Gibco; Thermo Fisher Scientific, Inc., Waltham, MA, USA), L-glutamine, $100 \mathrm{IU} / \mathrm{ml}$ penicillin, $100 \mathrm{mg} / \mathrm{ml}$ streptomycin (Invitrogen; Thermo Fisher Scientific, 
Inc.), sodium pyruvate and nonessential amino acids. All cells were cultured in a $5 \% \mathrm{CO}_{2}$ incubator at $37^{\circ} \mathrm{C}$.

PARK2 overexpression lentivirus and short hairpin RNA (shRNA) lentivirus construction. The human PARK2 overexpression lentivirus (containing the whole coding sequence; https://www.ncbi.nlm.nih.gov/nuccore/NM_004562.2) was purchased from Shanghai GenePharma Co., Ltd. (Shanghai, China). The blank vector lentivirus, acting as a control, an shRNA lentivirus targeting human PARK2 (shPARK2 forward, 5'-GATCCTCCAAAGAAACCATCAAGA ACT TCCTGTCAGATTCTTGATGGTTTCTTTGGATTTTTG-3' and reverse, 5'-AATTCAAAAATCCAAAGAAACCATCAA GAATCTGACAGGAAGTTCTTGATGGTTTCTTTGGAG G-3') and a scrambled shRNA lentivirus, acting as a negative control, were also designed and synthesized by Shanghai GenePharma Co., Ltd.

Small interfering RNA (siRNA) design and transfections. A172 cells and A172/shPARK2 cells were transfected with ZEB1 siRNA (siZEB1; sense, 5'-CAGUGUUCCAUGCUU AAGAdTdT-3' and anti-sense, 5'-UCUUAAGCAUGGAAC ACUGdTdT-3') and a negative non-targeted control siRNA (siControl sense, 5'-TTCTCCGAACGTGTCACGTdTdT-3' and anti-sense, 5'-ACGTGACACGTTCGGAGAAdTdT-3'), which were designed and synthesized by Shanghai GenePharma Co., Ltd. The cells were cultured until 30-50\% confluence was attained and then $2.0 \mu \mathrm{g}$ siRNA and $10.0 \mu \mathrm{l}$ Lipofectamine ${ }^{\circledR} 2000$ transfection reagent (Invitrogen; Thermo Fisher Scientific, Inc.) were separately diluted in serum-free Opti-MEM-1 medium (Gibco; Thermo Fisher Scientific, Inc.) and then mixed together. The mixture was subsequently incubated at room temperature for $20 \mathrm{~min}$ and then added directly onto the cells for $6 \mathrm{~h}$ at $37^{\circ} \mathrm{C}$.

RNA extraction and reverse transcription-quantitative polymerase chain reaction $(R T-q P C R)$. RNA was isolated from $1 \times 10^{6}$ U87 or A172 cells using TRIzol reagent (Sigma-Aldrich; Merck KGaA, Darmstadt, Germany) according to manufacturer's protocol. Equal quantities of RNA (500 ng) were reverse transcribed into cDNA using a QuantiTect reverse transcription kit according to the manufacturer's protocol (Qiagen Inc., Valencia, CA, USA). The resulting cDNA was used as the template for qPCR. Oligonucleotide primers were synthesized (Invitrogen; Thermo Fisher Scientific, Inc.), and qPCR was performed in a $20 \mu \mathrm{l}$ volume containing $2 \mu \mathrm{l}$ template cDNA, 2X SYBR-Green master mix (Roche Diagnostics $\mathrm{GmbH}$, Mannheim, Germany) and $10 \mathrm{pM}$ of each primer. The primer sequences were as follows: E-cadherin forward, 5'-TTGACG CCGAGAGCTACAC-3' and reverse, 5'-GTCGACCGGTGC AATCTT-3'; vimentin forward, 5'-TACAGGAAGCTGCTG GAAGG-3' and reverse, 5'-ACCAGAGGGAGTGAATCC AG-3'; and $\beta$-actin forward, 5'-TTGTTACAGGAAGTCCCT TGCC-3'; and reverse, 5'-ATGCTATCACCTCCCCTGTGT G-3'. Amplification was performed using the Light Cycler 480 PCR system (Roche Diagnostics $\mathrm{GmbH}$ ) under the following thermocycling conditions: $95^{\circ} \mathrm{C}$ for $30 \mathrm{sec}$ followed by 40 cycles of $95^{\circ} \mathrm{C}$ for $5 \mathrm{sec}$ and $60^{\circ} \mathrm{C}$ for $20 \mathrm{sec}$. Quantity values for gene expression were generated by the relative quantification $\left(2^{-\Delta \Delta \mathrm{Cq}}\right)$ method (11); fluorescence generated by each sample was normalized to the $\beta$-actin product for each gene of interest. The experiments were repeated three times.

Migration assay. The migration of U87 and A172 cells was assayed using 24-well collagen-coated Boyden chambers (Chemicon; EMD Millipore, Billerica, MA, USA) with $8 \mu \mathrm{m}$ pores (12). A total of $4 \times 10^{4}$ cells from indicated groups (NC and PARK2 or shControl and shPARK2) were seeded in the upper chamber $(0.2 \mathrm{ml}$ DMEM in the upper chamber) and $0.8 \mathrm{ml}$ DMEM with $10 \% \mathrm{FBS}$ was added in the lower chamber. Following an incubation period of $48 \mathrm{~h}$ at $37^{\circ} \mathrm{C}$, migrating cells were quantified according to the manufacturer's protocol. Briefly, the cells that migrated to the basal side of the membrane were fixed with $4 \%$ paraformaldehyde for $5 \mathrm{~min}$ at $25^{\circ} \mathrm{C}$ and then stained with $1 \%$ crystal violet for $10 \mathrm{~min}$ at $25^{\circ} \mathrm{C}$. The cells were subsequently visualized and photographed with a CKX41 light microscope (Olympus Corporation, Tokyo, Japan) at x200 magnification. Images of three random fields from three replicate wells were obtained and the number of migratory or invasive cells was counted.

Invasion assay. The invasion of U87 and A172 cells was also assayed using 24-well collagen-coated Boyden chambers (Chemicon; EMD Millipore) with $8 \mu \mathrm{m}$ pores. Following resuspension in $200 \mu \mathrm{l}$ serum-free DMEM, 6x10 487 and A172 cells were seeded on Matrigel-coated chamber inserts (0.2 $\mathrm{ml}$ DMEM in the upper chamber and $0.8 \mathrm{ml}$ DMEM with $10 \% \mathrm{FBS}$ in the lower chamber) and incubated at $37^{\circ} \mathrm{C}$ for $48 \mathrm{~h}$ (BD Biosciences, San Jose, CA, USA). Wells were subsequently washed with PBS. The cells that migrated to the basal side of the membrane were fixed with $4 \%$ paraformaldehyde for $5 \mathrm{~min}$ at $25^{\circ} \mathrm{C}$ and then stained with $1 \%$ crystal violet for $10 \mathrm{~min}$ at $25^{\circ} \mathrm{C}$. The cells were subsequently visualized and photographed with a CKX41 light microscope (Olympus Corporation) at $\mathrm{x} 200$ magnification. Images of three random fields from three replicate wells were obtained and migratory or invasive cells were counted.

Statistical analysis. GraphPad Prism version 5.0 for Windows (GraphPad Software Inc., San Diego, CA, USA) was applied for the statistical analyses. Results were expressed as the mean \pm standard error of the mean. A Student's t-test (unpaired) was used to evaluate the statistical significance of the results. $\mathrm{P}<0.05$ was considered to indicate a statistically significant difference.

\section{Results}

Overexpression of PARK2 mitigates metastasis and invasion of GBM cells. Since the invasion-metastasis cascade may induce mortality in patients with GBM, the present study determined whether PARK2 regulated GBM progression by influencing metastasis. To select appropriate cell lines for further functional examination, PARK2 mRNA expression was assessed in five GBM cell lines. Since PARK2 mRNA expression in U87 cells was lower than in other cell lines (Fig. 1A), stable overexpression of PARK2 mRNA in U87 cells was induced via lentiviral infection. Overexpression efficiency was confirmed by RT-qPCR (Fig. 1B). Transwell migration and Matrigel invasion chamber assays were used 
A
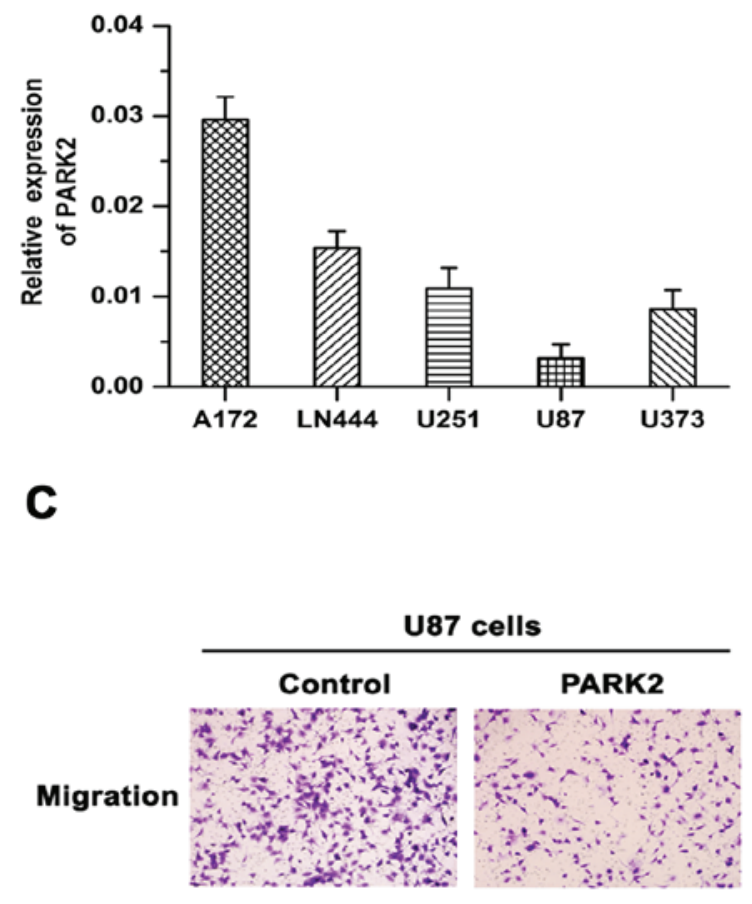

D

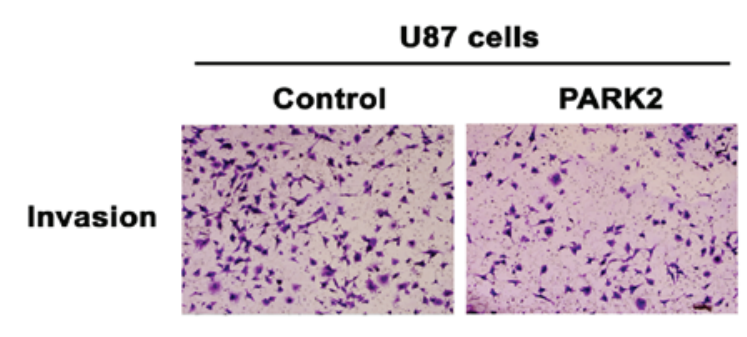

B
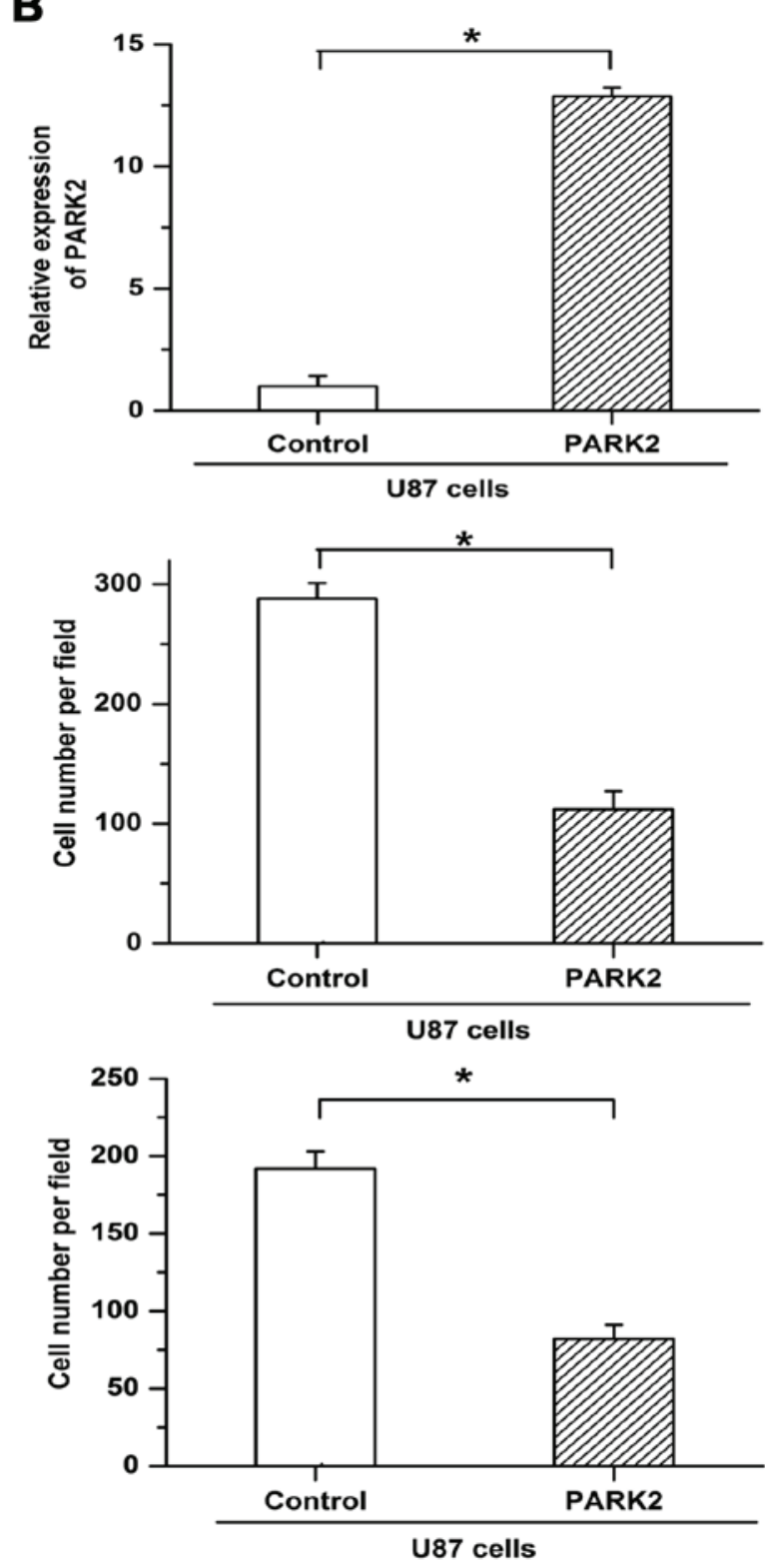

Figure 1. Overexpression of PARK2 decreased the migratory and invasive potential of GBM cells compared with normal PARK2 expression. (A) RT-qPCR was used to examine the expression of PARK2 mRNA in five GBM cell lines. (B) Efficiency of PARK2 overexpression was assessed using RT-qPCR in U87 cells. (C) Migration of U87 cells was attenuated by PARK2 overexpression compared with normal PARK2 expression. (D) Overexpression of PARK2 repressed invasion of U87 cells compared with normal PARK2 expression. Each experiment was performed in triplicate. Data were represented as the mean \pm standard error of the mean of three independent experiments (magnification, $\mathrm{x} 100$ ). ${ }^{*} \mathrm{P}<0.05$ vs. control. PARK2, Parkinson protein 2 E3 ubiquitin protein ligase; GBM, glioblastoma; RT-qPCR, reverse transcription-quantitative polymerase chain reaction.

to determine the effect of PARK2 on the metastasis of GBM cells. Migratory and invasive potential was revealed to be attenuated by PARK2 overexpression in U87 cells (Fig. 1C and D). The results suggested that overexpression of PARK2 repressed the metastasis of GBM cells.

PARK2 knockdown promotes migration and invasion of GBM cells. To reveal the function of PARK2 in the metastasis of GBM cells, shRNA was used to knock down expression of PARK2 in A172 cells. Knockdown efficiency was verified by RT-qPCR (Fig. 2A). Knockdown of PARK2 enhanced cell migration in A172 cells (Fig. 2B). Similar results were obtained in terms of cell invasion, with invasion being facilitated by silencing the expression of PARK2 (Fig. 2C). These results indicated that knockdown of PARK2 promoted cell metastasis and the progression of GBM.

Promotive effects of PARK2 knockdown on metastasis are reduced by silencing expression of $Z E B 1$. ZEB1 is a key regulator in the metastasis of tumor cells. Expression of ZEB1 is significantly upregulated in invasive glioma tissue (13). The present study assessed whether a regulatory relationship exists between PARK2 and ZEB1 in GBM cells. Expression of ZEB1 was significantly decreased following overexpression of PARK2, while inhibiting PARK2 expression via shRNA led to increased expression of ZEB1 (Fig. 3A and B). To verify the functions of ZEB1 in PARK2 knockdown-promoted metastasis, siRNA was used to repress the expression of ZEB1. Knockdown 

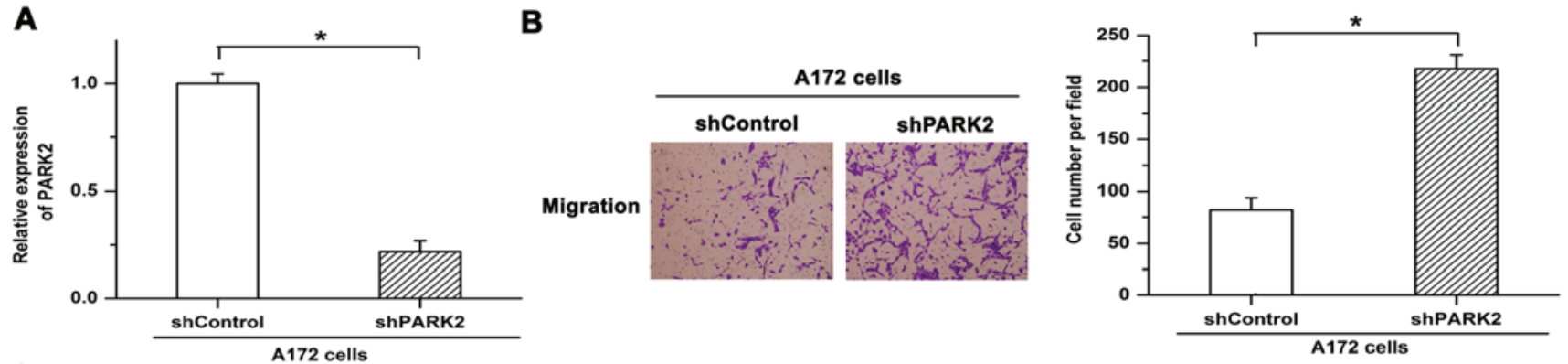

C
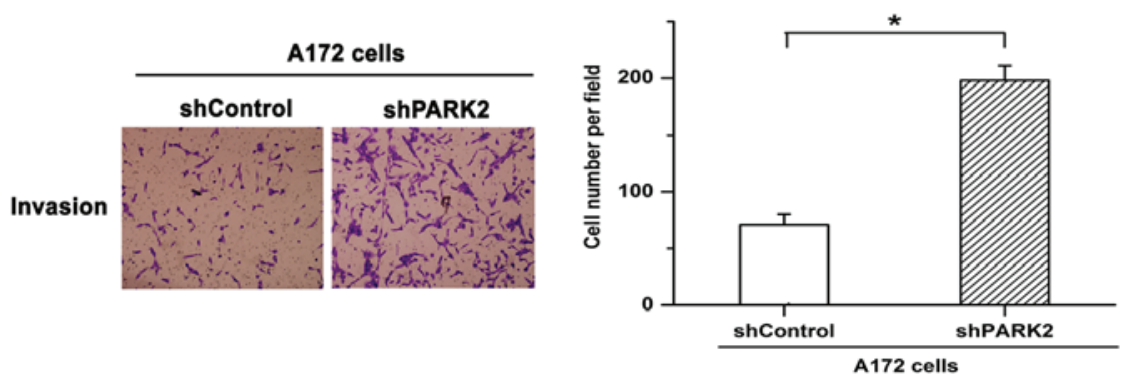

Figure 2. PARK2 knockdown increased migration and invasion of glioblastoma cells. (A) RT-qPCR revealed that PARK2 expression was significantly inhibited by PARK2 shRNA compared with control shRNA. (B) Migration was enhanced by PARK2 knockdown in A172 cells. (C) Knockdown of PARK2 promoted cell invasion in A172 cells. Data were represented as the mean \pm standard error of the mean of three independent experiments (magnification, $\mathrm{x} 100)$. "P<0.05 vs. control. PARK2, Parkinson protein 2 E3 ubiquitin protein ligase; RT-qPCR, reverse transcription-quantitative polymerase chain reaction; shRNA/sh, short hairpin RNA.

A

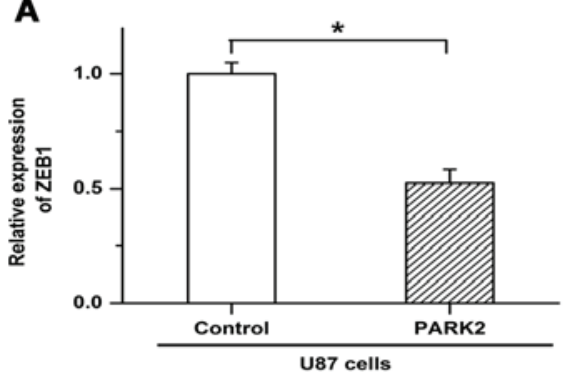

D

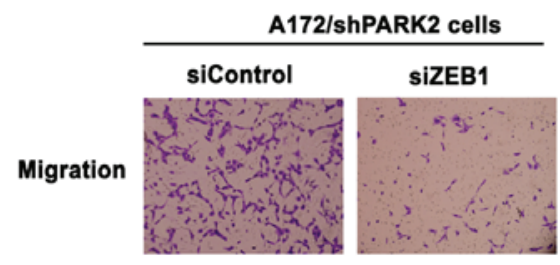

$\mathbf{E}$

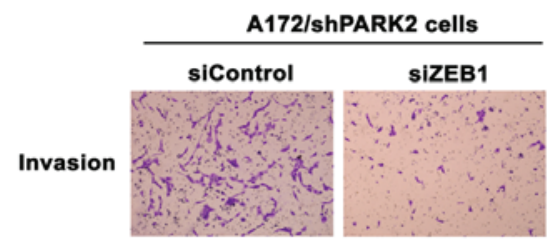

B

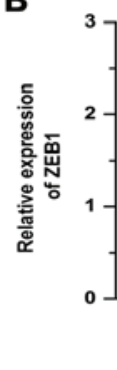

C

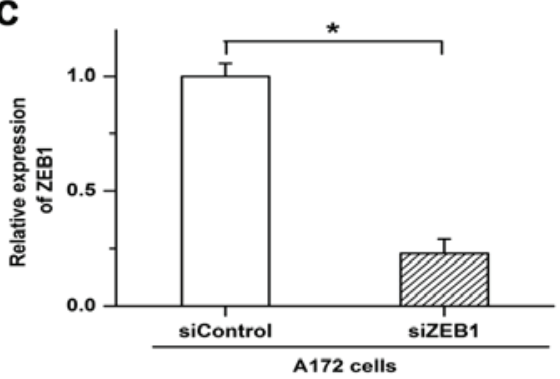

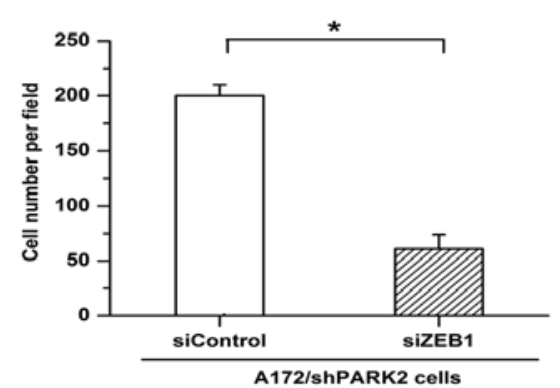

*

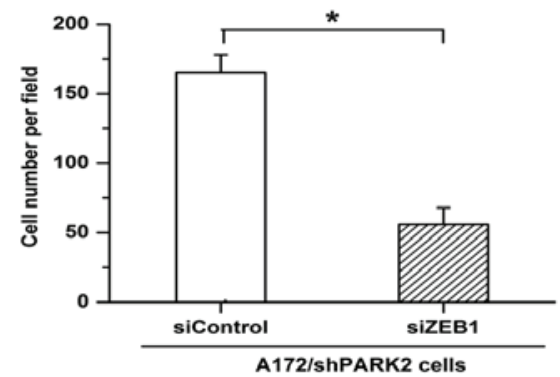

Figure 3. ZEB1 knockdown attenuated the promotive effects of PARK2 knockdown on metastasis in glioblastoma cells. (A) Overexpression of PARK2 repressed the expression of ZEB1 in U87 cells compared with normal PARK2 expression. (B) Expression of ZEB1 was increased by PARK2 knockdown in A172 cells. (C) Knockdown efficiency of siZEB1 was assessed using RT-qPCR. (D) PARK2 knockdown-facilitated cell migration and (E) invasion was antagonized by siZEB1. Data were represented as the mean \pm standard error of the mean of three independent experiments (magnification, $\mathrm{x} 100)$. ${ }^{*} \mathrm{P}<0.05$ vs. control. ZEB1, zinc finger E-box-binding homeobox 1; PARK2, Parkinson protein 2 E3 ubiquitin protein ligase; si, small interfering RNA; RT-qPCR, reverse transcription-quantitative polymerase chain reaction; sh, short hairpin RNA. 
A

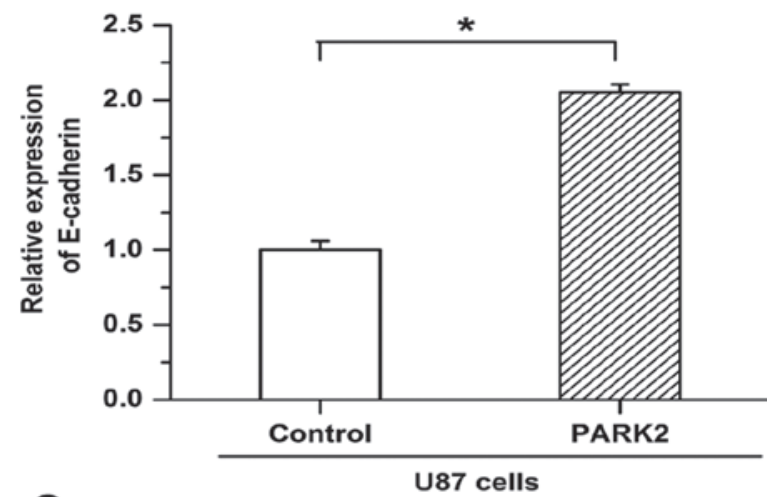

C

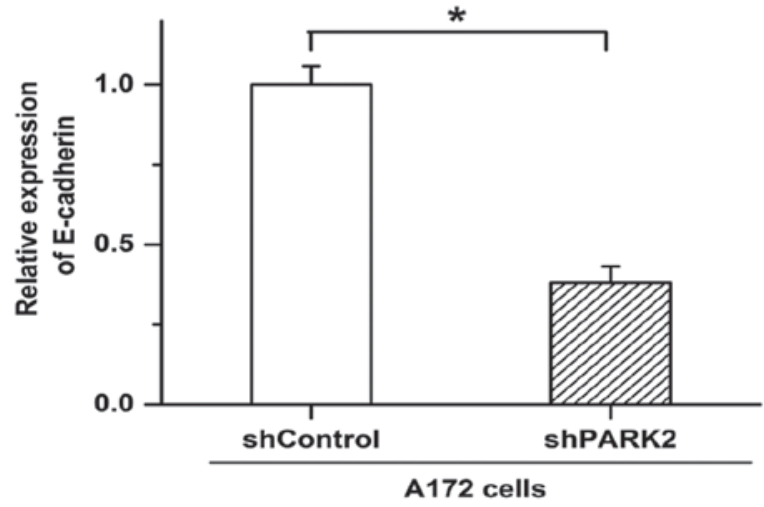

E

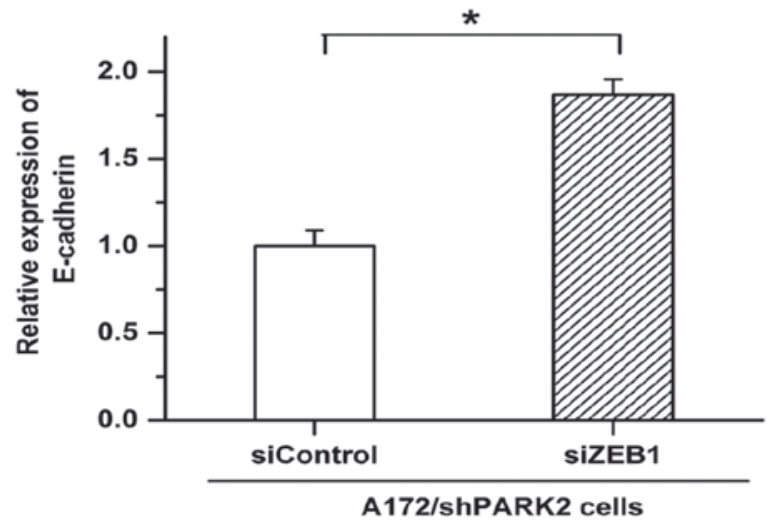

B
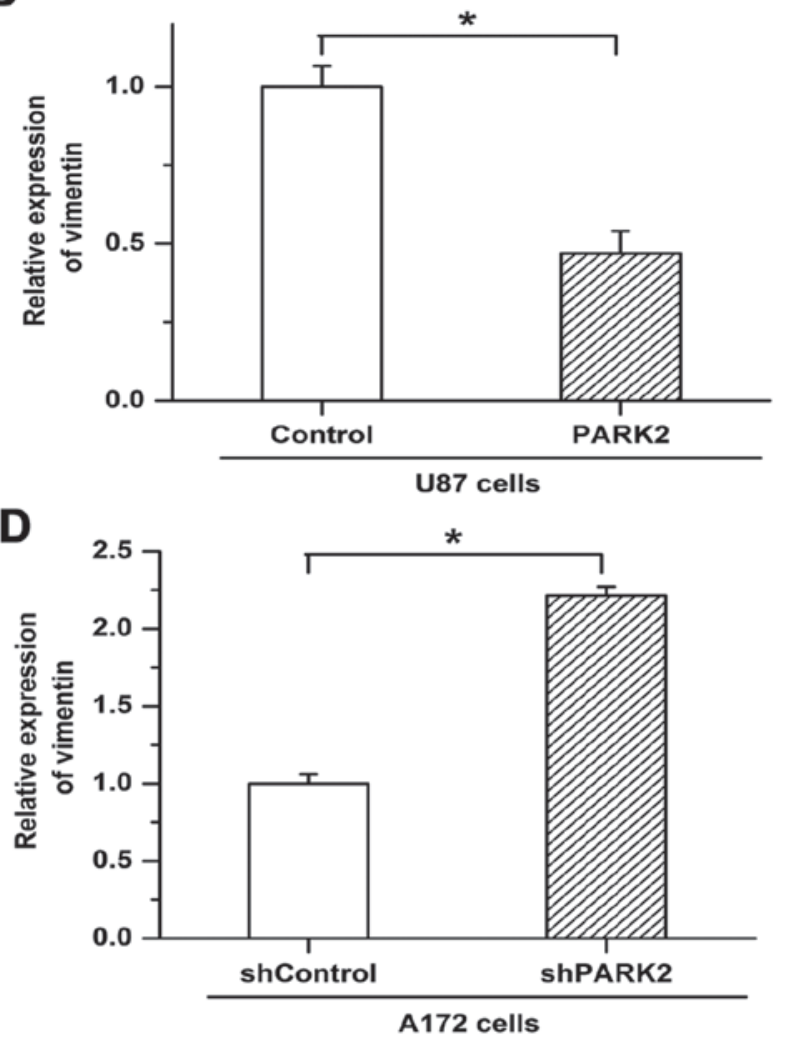

F

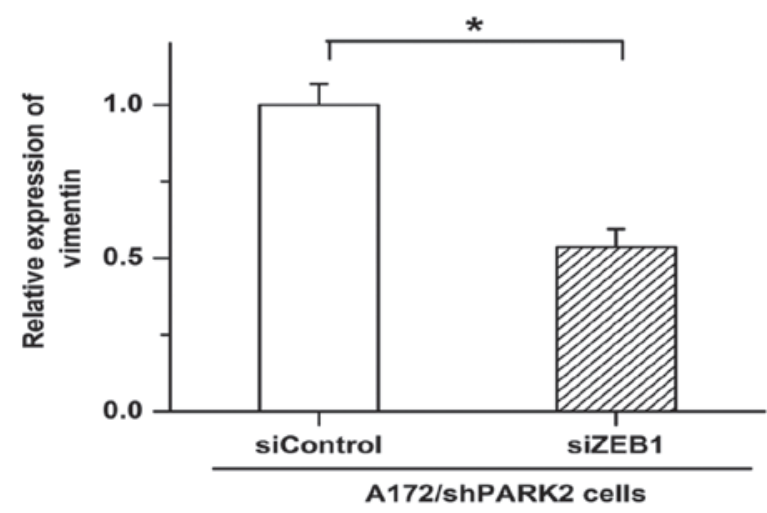

Figure 4. Effects of PARK2 on EMT were mediated by ZEB1 in glioblastoma cells. (A) Compared with normal PARK2 expression, PARK2 overexpression increased E-cadherin expression and (B) decreased vimentin expression. (C) PARK2 knockdown increased vimentin expression and (D) decreased E-cadherin expression in A172 cells. (E) Regulatory effects of PARK2 knockdown on the expression of E-cadherin and (F) vimentin were mitigated by siZEB1. Data were represented as the mean \pm standard error of the mean of three independent experiments. * P $<0.05$ vs. control. PARK2, Parkinson protein 2 E3 ubiquitin protein ligase; EMT, epithelial-mesenchymal transition; ZEB1, zinc finger E-box-binding homeobox 1; E-cadherin, epithelial cadherin; si, small interfering RNA; sh, short hairpin RNA.

efficiency of siZEB1 was demonstrated by RT-qPCR (Fig. 3C). Knockdown of PARK2-facilitated cell migration and invasion was eliminated by siZEB1 (Fig. 3D and E). These results demonstrated that ZEB1 serves as an important mediator in PARK2-regulated GBM cell metastasis.

PARK2-regulated EMT is mediated by ZEB1 in GBM cells. EMT is associated with tumor metastasis, and chemotherapy resistance is more frequently observed in cancer cells undergoing EMT (14). The present study revealed the involvement of PARK2 in EMT. Expression of the EMT markers epithelial cadherin (E-cadherin) and vimentin was examined by
RT-qPCR. PARK2 overexpression increased the expression of E-cadherin in U87 cells but was associated with decreased expression of vimentin (Fig. 4A and B). Knockdown of PARK2 in A172 cells repressed the expression of E-cadherin and induced the expression of vimentin (Fig. 4C and D). Furthermore, decreased expression of E-cadherin and increased expression of vimentin, as triggered by PARK2 knockdown, were reversed by ZEB1 siRNA, with E-cadherin expression increasing and vimentin expression decreasing compared with siControl (Fig. 4E and F). These results suggested that PARK2 negatively regulated EMT by depressing ZEB1 expression in glioma cells. 


\section{Discussion}

GBM is one of the most aggressive human malignancies $(1,2)$. However, current treatment strategies are ineffective and the pathogenesis of GBM and the corresponding molecular mechanisms are not yet fully understood. Therefore, results potentially leading to novel therapeutic targets of GBM are vital. The results of the present study suggested that PARK2 repressed the metastasis and invasion of GBM cells and that PARK2 negatively regulated EMT by depressing ZEB1 expression. These results demonstrated a the involvement of PARK2 in suppressing the metastasis and invasion of GBM cells.

Crucially, the present study identified PARK2 as a tumor suppressor in GBM cells. Previous studies have demonstrated that somatic alterations to PARK2 are frequently observed in numerous types of human tumor. Deficiency of PARK2 in transgenic mice results in colorectal adenoma and hepatocellular carcinoma occurring more frequently $(6,7)$. Furthermore, restoring PARK2 expression depresses the proliferation of cancer cells derived from brain, breast, and lung tissue $(12,15)$. However, the function of PARK2 in the metastasis of GBM and the associated molecular mechanisms are not yet fully understood. The present study demonstrated that PARK2 overexpression mitigated the metastasis and invasion of GBM cells. Conversely, migration and invasion of cancer cells were facilitated by knockdown of PARK2. These results suggested that PARK2 functioned as a tumor suppressor during the metastasis of GBM cells.

EMT is key in the initiation of metastasis in cancer cells (16). Cancer cells undergoing EMT are more resistant to radiotherapy and are able to acquire stem cell traits $(17,18)$. EMT, a reversible process, is characterized by the loss of polarized features, the movement away from neighboring cells and increased motility and invasion, contributing to a disassembly of cell-cell junctions. EMT is also characterized by decreased expression of E-cadherin and increased expression of mesenchymal molecular markers, including vimentin (19-21). ZEB1 induces EMT, and ZEB1 expression is associated with the survival and therapy response of patients with tumors (22). Previous studies have demonstrated that silencing ZEB1 expression hampers metastasis and invasion in diverse types of human cancer; ZEB1 is therefore a potential therapeutic target for repressing the development of tumors $(23,24)$. The present study suggested that PARK2 has regulatory effects on the expression of ZEB1 and EMT. The present study demonstrated that the overexpression of PARK2 significantly repressed the expression of ZEB1, while PARK2 knockdown resulted in increased expression of ZEB1. The promotive effects of PARK2 knockdown on metastasis were reduced by silencing expression of ZEB1. Furthermore, PARK2 overexpression blocked the process of EMT, which was represented as the upregulation of E-cadherin and downregulation of vimentin. Conversely, knockdown of PARK2 induced the expression of vimentin and repressed E-cadherin expression in A172 cells, and the effects of PARK2 knockdown on EMT were attenuated by siZEB1. These results suggested that PARK2 negatively regulated EMT by depressing ZEB1 expression in GBM cells.

In conclusion, the results of the present study suggested that PARK2 mitigated the metastasis and invasion of GBM cells and inhibited the progression of GBM by functioning as a tumor suppressor. Furthermore, ZEB1 was an important mediator in PARK2-suppressed the metastasis and EMT in GBM. The present study elucidated an important underlying mechanism regulating the metastasis and invasion of GBM cells, and provided a potential therapeutic approach for GBM.

\section{Acknowledgements}

The present study was supported by the Natural Science Foundation of Heilongjiang Province (grant no. H201434), the Postdoctoral Foundation of Heilongjiang Province (grant no. LBH-Z11096) and the National International Science and Technology Cooperation Foundation of China (grant no. 2014DFA31630).

\section{References}

1. Holdhoff $\mathrm{M}$ and Grossman SA: Controversies in the adjuvant therapy of high-grade gliomas. Oncologist 16: 351-358, 2011.

2. Vehlow $A$ and Cordes N: Invasion as target for therapy of glioblastoma multiforme. Biochim Biophys Acta 1836: 236-244, 2013.

3. Montana V and Sontheimer $\mathrm{H}$ : Bradykinin promotes the chemotactic invasion of primary brain tumors. J Neurosci 31: 4858-4867, 2011.

4. Stupp R, Hegi ME, Gilbert MR and Chakravarti A: Chemoradiotherapy in malignant glioma: Standard of care and future directions. J Clin Oncol 25: 4127-4136, 2007.

5. $\mathrm{Xu} \mathrm{L}$, Lin DC, Yin D and Koeffler HP: An emerging role of PARK2 in cancer. J Mol Med (Berl) 92: 31-42, 2014.

6. Poulogiannis G, McIntyre RE, Dimitriadi M, Apps JR, Wilson CH, Ichimura K, Luo F, Cantley LC, Wyllie AH, Adams DJ and Arends MJ: PARK2 deletions occur frequently in sporadic colorectal cancer and accelerate adenoma development in Apc mutant mice. Proc Natl Acad Sci USA 107: 15145-15150, 2010

7. Fujiwara M, Marusawa H, Wang HQ, Iwai A, Ikeuchi K, Imai Y, Kataoka A, Nukina N, Takahashi R and Chiba T: Parkin as a tumor suppressor gene for hepatocellular carcinoma. Oncogene 27: 6002-6011, 2008.

8. Tay SP, Yeo CW, Chai C, Chua PJ, Tan HM, Ang AX, Yip DL, Sung JX, Tan PH, Bay BH, et al: Parkin enhances the expression of cyclin-dependent kinase 6 and negatively regulates the proliferation of breast cancer cells. J Biol Chem 285: 29231-29238, 2010.

9. Picchio MC, Martin ES, Cesari R, Calin GA, Yendamuri S, Kuroki T, Pentimalli F, Sarti M, Yoder K, Kaiser LR, et al: Alterations of the tumor suppressor gene Parkin in non-small cell lung cancer. Clin Cancer Res 10: 2720-2724, 2004.

10. Veeriah S, Taylor BS, Meng S, Fang F, Yilmaz E, Vivanco I, Janakiraman M, Schultz N, Hanrahan AJ, Pao W, et al: Somatic mutations of the Parkinson's disease-associated gene PARK2 in glioblastoma and other human malignancies. Nat Genet 42: 77-82, 2010.

11. Livak KJ and Schmittgen TD: Analysis of relative gene expression data using real-time quantitative PCR and the 2(-Delta Delta C(T)) Method. Methods 25: 402-408, 2001.

12. Pardo A, Gibson K, Cisneros J, Richards TJ, Yang Y, Becerril C, Yousem S, Herrera I, Ruiz V, Selman M and Kaminski N: Up-regulation and profibrotic role of osteopontin in human idiopathic pulmonary fibrosis. PLoS Med 2: e251, 2005.

13. Zhang L, Zhang W, Li Y, Alvarez A, Li Z, Wang Y, Song L, Lv D, Nakano I, Hu B, et al: SHP-2-upregulated ZEB1 is important for PDGFR $\alpha$-driven glioma epithelial-mesenchymal transition and invasion in mice and humans. Oncogene 35: 5641-5652, 2016.

14. Siebzehnrubl FA, Silver DJ, Tugertimur B, Deleyrolle LP, Siebzehnrubl D, Sarkisian MR, Devers KG, Yachnis AT, Kupper MD, Neal D, et al: The ZEB1 pathway links glioblastoma initiation, invasion and chemoresistance. EMBO Mol Med 5: 1196-1212, 2013.

15. Yeo CW, Ng FS, Chai C, Tan JM, Koh GR, Chong YK, Koh LW, Foong CS, Sandanaraj E, Holbrook JD, et al: Parkin pathway activation mitigates glioma cell proliferation and predicts patient survival. Cancer Res 72: 2543-2553, 2012. 
16. Sun X, Liu M, Hao J, Li D, Luo Y, Wang X, Yang Y, Li F, Shui W, Chen Q and Zhou J: Parkin deficiency contributes to pancreatic tumorigenesis by inducing spindle multipolarity and misorientation. Cell Cycle 12: 1133-1141, 2013.

17. Tsai JH and Yang J: Epithelial-mesenchymal plasticity in carcinoma metastasis. Genes Dev 27: 2192-2206, 2013.

18. Wu KJ and Yang MH: Epithelial-mesenchymal transition and cancer stemness: The Twist1-Bmil connection. Biosci Rep 31: 449-455, 2011.

19. Guo Z, Hardin H and Lloyd RV: Cancer stem-like cells and thyroid cancer. Endocr Relat Cancer 21: T285-T300, 2014.

20. Yao D, Dai C and Peng S: Mechanism of the mesenchymal-epithelial transition and its relationship with metastatic tumor formation. Mol Cancer Res 9: 1608-1620, 2011.

21. von Gise A and Pu WT: Endocardial and epicardial epithelial to mesenchymal transitions in heart development and disease. Circ Res 110: 1628-1645, 2012.
22. Yamada S, Okumura N, Wei L, Fuchs BC, Fujii T, Sugimoto H, Nomoto S, Takeda S, Tanabe KK and Kodera Y: Epithelial to mesenchymal transition is associated with shorter disease-free survival in hepatocellular carcinoma. Ann Surg Oncol 21: 3882-3890, 2014.

23. Gibbons DL, Lin W, Creighton CJ, Rizvi ZH, Gregory PA, Goodall GJ, Thilaganathan N, Du L, Zhang Y, Pertsemlidis A and Kurie JM: Contextual extracellular cues promote tumor cell EMT and metastasis by regulating miR-200 family expression. Genes Dev 23: 2140-2151, 2009.

24. Chu K, Boley KM, Moraes R, Barsky SH and Robertson FM: The paradox of E-cadherin: Role in response to hypoxia in the tumor microenvironment and regulation of energy metabolism. Oncotarget 4: 446-462, 2013. 